\title{
Irisin in the primate hypothalamus and its effect on GnRH in vitro
}

\author{
Fazal Wahab1, Ikram Ullah Khan², Ignacio Rodriguez Polo1,3, Hira Zubair2, Charis Drummer1,3, Muhammad Shahab² \\ and Rüdiger Behr1,3
}

1Platform Degenerative Diseases, German Primate Center, Leibniz Institute for Primate Research, Göttingen, Germany

2Department of Animal Sciences, Faculty of Biological Sciences, Quaid-i-Azam University, Islamabad, Pakistan

${ }^{3}$ DZHK (German Center for Cardiovascular Research), Partner Site Göttingen, Göttingen, Germany

Correspondence should be addressed to F Wahab or R Behr: fwahab@gmail.com or rbehr@dpz.eu

\begin{abstract}
Irisin, encoded by the FNDC5 gene, is a recently discovered endocrine factor mainly secreted as a myokine and adipokine. However, irisin/FNDC5 expression has also been reported in different other organs including components of the reproductive axis. Yet, there is the scarcity of data on $F N D C 5$ /irisin expression, regulation and its reproductive effects, particularly in primates. Here, we report the expression of FNDC5/irisin, along with PGC1A (peroxisome proliferator-activated receptor gamma coactivator 1-alpha) and ERRA (estrogen-related receptor alpha), in components of the reproductive axis of marmoset monkeys. Hypothalamic FNDC5 and ERRA transcript levels are developmentally regulated in both male and female. We further uncovered sex-specific differences in FNDC5, ERRA and PGC1A expression in muscle and the reproductive axis. Moreover, irisin and ERR $\alpha$ co-localize in the marmoset hypothalamus. Additionally, in the arcuate nucleus of rhesus monkeys, the number of irisin+ cells was significantly increased in short-term fasted monkeys as compared to ad libitum-fed monkeys. More importantly, we observed putative interaction of irisin-immunoreactive fibers and few GnRH-immunoreactive cell bodies in the mediobasal hypothalamus of the rhesus monkeys. Functionally, we noted a stimulatory effect of irisin on GnRH synthesis and release in mouse hypothalamic neuronal GT1-7 cells. In summary, our findings show that FNDC5 and irisin are developmentally, metabolic-status dependently and sex-specifically expressed in the primate hypothalamic-pituitary-gonadal axis and exert a stimulatory effect on $\mathrm{GnRH}$ expression and release in mouse hypothalamic cells. Further studies are required to confirm the reproductive effects of irisin in vivo and to illuminate the mechanisms of its regulation.
\end{abstract}

Journal of Endocrinology (2019) 241, 175-187

\section{Introduction}

The gonadal function is controlled by a complex interaction of regulatory signals, involving hypothalamus, pituitary and the gonads themselves. Altogether, they form the reproductive axis (a.k.a. the hypothalamicpituitary-gonadal (HPG) axis) (Wahab et al. 2011a, 2015, 2016a, Plant 2015a, Corradi et al. 2016). In this axis, pulsatile release of the hypothalamic gonadotropinreleasing hormone $(\mathrm{GnRH})$ regulates the secretion of pituitary gonadotropins, that is luteinizing hormone (LH) and follicle-stimulating hormone (FSH), which play a key role in the control of gonadal maturation and functions (Clarke 2011, Wahab et al. 2011a, Plant 2015a). 
The reproductive axis activities change during postnatal development, adulthood, pregnancy and aging. Shortly after birth, the reproductive axis activity starts at minipuberty and remains high during the infantile period in primates including humans (Terasawa \& Fernandez 2001, Plant 2015b). However, the reproductive axis activity in childhood and during the juvenile period is at nadir. At the time of true puberty, the reproductive axis resumes activity (Terasawa \& Fernandez 2001, Plant $2015 b$ ) and remains high during the active phase of the adult reproductive life. However, the reproductive axis performance in adults is sensitive to the current nutritional status of the body, environmental cues and the systemic steroid milieu in both male and female (Winter et al. 1987, Clarke \& Caraty 2013, Wahab et al. 2013, 2015, Henningsen et al. 2016, Evans \& Anderson 2017). In particular, in the female, the reproductive axis activity is altered during various phases of the reproductive cycle and pregnancy (Beshay \& Carr 2013, Castellano \& Tena-Sempere 2016). Pregnancy is characterized by the attenuation of the GnRH-pituitary-gonadotropic axis (Al-Gubory et al. 2003). Nevertheless, the exact mechanism of the alterations in the reproductive axis activity in response to internal and external cues during postnatal development, pregnancy and aging is still ill defined.

A recent potential candidate for linking metabolic reserves, especially body weight (protein mass)-related information with the awakening of the reproductive axis at the time of puberty onset and regulation of adult reproduction, is irisin (Wahab et al. 2016b). Irisin is a recently discovered peptide hormone mainly secreted by muscle and adipose tissue (Boström et al. 2012, Roca-Rivada et al. 2013, Perakakis et al. 2017). Irisin is encoded by the FNDC5 (fibronectin type III domaincontaining protein 5) gene. The FNDC5 gene encodes a precursor FNDC5 transmembrane protein (human, 212; mouse and rat, 209 amino acids). This precursor FNDC5 protein is cleaved by an unknown protease, and its extracellular domain is shed into systemic circulation as irisin (Boström et al. 2012, Perakakis et al. 2017). Irisin is a 112 amino acid peptide. Very recently, the irisin receptor, $\alpha \mathrm{V} / \beta 5$ integrin, has been discovered in adipose tissues and bone (Kim et al. 2018). The transcriptional coactivator peroxisome proliferator-activated receptor gamma coactivator 1-alpha (PGC1 $\alpha$ ) and estrogen-related receptor alpha $(E R R \alpha)$, an orphan nuclear receptor and transcription factor, are involved in the regulation of Fndc5 expression in various tissues (Boström et al. 2012, Wrann et al. 2013).
Irisin has been implicated in the regulation of fat and energy metabolism (Rodríguez et al. 2016). Importantly, energy metabolism is closely linked to reproductive function (Wahab et al. 2013, 2015). The majorities of molecular factors that participate in energy homeostasis also modulate reproductive function directly or indirectly via both central hypothalamic and direct gonadal actions (Wade \& Jones 2004, Crown et al. 2007, Wahab et al. 2013, 2015, 2018, Castellano \& Tena-Sempere 2016, Evans \& Anderson 2017). Indeed, in light of recent studies (Aydin et al. 2014, Poretsky et al. 2017), irisin is a novel candidate factor for the modulation or even regulation of reproductive function. More importantly, it has been reported that expression of Fndc5 transcripts increases in the mouse brain during different postnatal developmental stages (Wrann et al. 2013). Furthermore, plasma levels of irisin change during various stages of puberty in humans (Hofmann et al. 2014, Reinehr et al. 2015). On the basis of these observations and previous findings of critical body weight (body fat and protein mass) for puberty commencement (Frisch \& Revelle 1970, Baker 1985), we recently proposed a hypothesis that irisin might serve as a metabolic trigger for puberty onset (Wahab et al. 2016b). We also proposed that FNDC5 and irisin might be expressed and/or act on peripheral and central tissues involved in the regulation of reproduction. Therefore, this study was planned to illuminate details of FNDC5 mRNA and irisin expression in the different anatomical and functional components of the reproductive axis - especially alterations in their expression at various postnatal developmental stages and to unveil a potential role of irisin in the hypothalamic modulation of reproductive axis activities in mammals. Different sets of experiments were used to study the potential roles of irisin in the regulation of reproductive function in the common marmoset monkey and rhesus monkey as representative of primates. Moreover, the effect of irisin on GnRH synthesis and release in the mouse hypothalamic GT1-7 cell was also studied.

\section{Materials and methods}

\section{Marmoset monkey hypothalamic tissue samples}

For this study, hypothalami or whole brains of NB, infantile, juvenile, pubertal and adult animals were analyzed. These tissue samples were obtained from the primate tissue banks of the Deutsches Primatenzentrum (DPZ), Göttingen, Germany. Samples were obtained for instance under license numbers 42502-04-066/06, 42502-04-10/0063 and 42502-04-14/1462 (the primary purposes of these studies were different from the one of 
the present study, but euthanasia of the animals allowed the collection of the samples). Additionally, some animals were killed on purpose for tissue isolation, which is possible without specific permission ('Nicht als Tierversuch gilt das Töten eines Tieres, soweit dies ausschließlich erfolgt, um dessen Organe oder Gewebe zu wissenschaftlichen Zwecken zu verwenden'.; § 7 Abs. 2 Nr.3 Deutsches Tierschutzgesetz. Content translation: Killing of an animal is not subject to authorization if this is done only for the purpose of an organ or tissue isolation for scientific purposes without any experimentation on the animal prior to euthanasia). Plausible scientific reasons for euthanasia were documented according to the official regulations, and the regulatory office was informed at the end of the year. The DPZ is registered and authorized by the local and regional veterinary governmental authorities for primate breeding and research (Reference number 122910.3311900, PK Landkreis Göttingen). For dissecting the hypothalamus out of the monkey brain, two coronal and two parasagittal cuts were carried out. The parasagittal cuts about $2.5 \mathrm{~mm}$ from midline were done at the lateral side of the hypothalamus and coronal cuts at the level of mammillary bodies and caudal to the optic chiasm. Furthermore, the hypothalamus was subsequently divided into two hemi-hypothalami. One of the hemi-hypothalami was used for real-time quantitative polymerase chain reaction (qPCR) analysis. The anatomy of the common marmoset brain was determined according to a marmoset brain atlas (Palazzi \& Bordier 2008).

The rhesus monkey study was conducted in the Primate Facility of the Quaid-I-Azam University, Islamabad, Pakistan. The hypothalamic tissues were taken from four adult monkeys (5-7 years old). Out of these four monkeys, two monkeys were normal fed and two were $48 \mathrm{~h}$ fasted at the time of euthanasia. The animals were killed after intramuscular (IM) injection of ketamine hydrochloride (Ketler, Astarapin, Germany; $10 \mathrm{mg} / \mathrm{kg} \mathrm{BW}$ IM) and followed by an intravenous injection of ketamine (3-5 mL). Immediately, the brain was separated from the cranium. The hypothalami (mediobasal hypothalamus $(\mathrm{MBH})$ ) were then harvested from the brain as previously described (Wahab et al. 2011b). This study was approved by the departmental animal research ethics committee.

\section{Experimental design}

\section{FNDC5 and irisin expression in reproductive axis components}

The expression profiles of FNDC5, PGC-1A and ERRA mRNAs were checked and compared in adult male $(n=3-4)$ and female ( $n=3-4)$ hypothalami, pituitaries and gonads. The hypothalamic expression profiles of FNDC5, PGC-1A and ERRA mRNAs were also compared with neonatal (NB, 1-3 days), infantile (2 months), juvenile (5 months), pubertal (15 months) and adult ( $>5$ years) in both female and male monkeys. The cDNA for this study was utilized as collected previously (Wahab et al. 2017). Furthermore, immunohistochemical detection of irisin was carried out in NB female, adult male and female marmoset hypothalamic or whole brain sections. The adult female monkeys were in the follicular phase of the ovarian cycle.

Moreover, FNDC5, PGC-1A and ERRA mRNA expression profiles were also checked in the anterior and posterior hypothalami of both male and female monkeys at NB and adult stages ( $n=4$ /group). For this experiment, the hypothalamus was divided into anterior and posterior parts in the middle as reported previously (Wahab et al. 2017).

\section{Irisin expression and interaction with GnRH neurons in rhesus monkeys hypothalamus}

Double-fluorescence immunocytochemistry for irisin and GnRH neurons was performed on free-floating hypothalamic sections to examine the interaction between irisin and GnRH immunoreactivities in the rhesus monkey hypothalamic MBH. After isolation, a solution of $4 \%$ paraformaldehyde in $0.1 \mathrm{M}$ PBS ( $\mathrm{pH} 7.4$ ) was used for the fixation of hypothalamic blocks for $14-18 \mathrm{~h}$ at $4^{\circ} \mathrm{C}$. After fixation, blocks were first transferred to $20 \%$ sucrose solution (Fisher Chemical, Loughborough, UK). As the blocks sank to the bottom, these were transferred to $30 \%$ sucrose solution at $4^{\circ} \mathrm{C}$ until they sank in it. Lastly, consecutive $20 \mu \mathrm{m}$ thick sections anterior to posterior all over the whole hypothalamic block were cut on a cryostat (Bright OTF 5000; Bright Instrument Company, Huntingdon, UK) and preserved in cryo-preservative solution (1\% polyvinylepyrrolidone, 30\% ethylene glycol and $30 \%$ sucrose in PBS) at $-20^{\circ} \mathrm{C}$ until used for immunocytochemistry.

Testing the effect of irisin on GnRH1 expression and $\mathrm{GnRH}$ release in the mouse hypothalamic GT1-7 cell

The mouse hypothalamic GT1-7 cells (Mellon et al. 1990) were grown in Dulbecco's modified Eagle's medium (DMEM; Mediatech Inc., Herndon, VA, USA) supplemented with $10 \%$ heat-inactivated fetal bovine serum (Hyclone, Logan, UT, USA), 100 U/mL penicillin and $100 \mathrm{mg} / \mathrm{mL}$ streptomycin (Gibco) in an atmosphere 
with $5 \% \mathrm{CO}_{2}$ at $37^{\circ} \mathrm{C}$. Four-hour serum-starved cells were treated with irisin (Phoenix Pharmaceutical Inc., San Diego, CA, USA) at final concentrations of $0.1,1$ and $10 \mathrm{nM}$ for 15 and $60 \mathrm{~min}$ in serum-free condition. Control wells were exposed in parallel to the vehicle (PBS) and are referred to as non-treated groups (NT). GnRH was measured using the EIA GnRH kit (Phoenix Pharmaceutical) according to the manufacturer's recommendations.

\section{Immunohistochemistry on sectioned tissues}

Immunohistochemical (IHC) staining of whole hypothalamic/brain sections was carried out as mentioned previously (Wahab et al. 2017). After collection, hypothalamic tissues were kept overnight in Bouin's solution for fixation. After at least 2 days of washes in 70\% EtOH, the tissues were embedded in paraffin and $5 \mu \mathrm{m}$ thick sections were cut using a microtome. These sections were mounted on slides. After deparaffinization and rehydration of the tissue sections, antigen retrieval was performed by cooking the tissue sections in a microwave oven in $10 \mathrm{mM}$ citrate buffer for $7 \mathrm{~min}$. When the tissue sections were cooled down, they were washed for $5 \mathrm{~min}$ in wash buffer. Subsequently, the blocking of endogenous peroxidase with a peroxidase blocking reagent (DakoCytomation, LSAB+ system-HRP, K0679) was done. The irisin antibody was purchased from Phoenix Pharmaceutical (Cat\# H-067-17). The dilution (1:1000) of the antibody was done in Tris-buffered saline plus 5\% bovine serum albumin (BSA). This antibody has been previously characterized and reported to detect irisin in the stomach, muscle and brain (Dun et al. 2013, Barja-Fernández et al. 2016) by western blotting and immunohistochemistry. In all incubation steps, the slides were placed horizontally in a humid plastic chamber. The incubation of tissue sections with the primary antibody was carried out overnight at $4^{\circ} \mathrm{C}$ while other incubations steps were performed at room temperature. Primary antibody on tissue sections was detected using a biotinylated second antibody polymer and horse-radish peroxidase (HRP)conjugated streptavidin from Dako (DakoCytomation Universal LSAB Plus kit). The chromogenic substrate for HRP was 3,3'-diaminobenzidine. Mayer's hematoxylin was used for counterstaining of tissues sections. Non-specific rabbit immunoglobulin G (IgG, dilution 1:1000) was used for control staining. The CRI Nuance multispectral imaging camera in conjunction with the Zeiss Axioskop microscope was used to capture images. Double IHC was performed using the Dako EnVision Doublestain System
(K5361) according to the manufacturer's instructions. Irisin antiserum was used in a 1:750 dilution and ERR $\alpha$ in a 1:800 dilution.

\section{Double-fluorescence immunocytochemistry}

Fluorescence double-staining for irisin and GnRH neurons was performed on free-floating hypothalamic sections to examine possible interaction between irisin and $\mathrm{GnRH}$ immunoreactivities. For irisin expression, a polyclonal primary irisin antibody raised in rabbit against human irisin (catalog no. H-067-17; Phoenix Pharmaceuticals, Inc.) was used. Alexa Fluor 488 goat anti-rabbit (catalog no. ab150077; Abcam) was used as a secondary antibody. For GnRH expression, a monoclonal primary antibody raised in mouse against human GnRH (Catalog no. sc-32292; Santa Cruz Biotechnology, Inc.) was used and Texas red-conjugated goat anti-mouse IgG-TR (Catalog no. sc- 2781; Santa Cruz Biotechnology, Inc.) was used as a secondary antibody.

From each animal, three random sections of the hypothalamus were obtained for irisin-GnRH dual labeled immunostaining and one section from each animal was processed as a primary antibody-omitted control. The hypothalamic sections were washed in phosphate buffered saline (PBS pH 7.3) (Omnipur PBS tablets; Calbiochem, EMD Chemicals Inc.) for $8 \times 15 \mathrm{~min}$ at room temperature.

Sections were incubated in a solution containing $0.03 \%$ Triton X-100 and $0.1 \%$ BSA in PBS to increase permeability and 10\% normal goat serum to block nonspecific binding or background staining. Incubation was done in a plate on a shaker (Serono Diagnostics, Merck $\mathrm{KGaA}$ ) for $120 \mathrm{~min}$ at room temperature. The sections were then incubated at $4^{\circ} \mathrm{C}$ for $48 \mathrm{~h}$ on a shaker in a cocktail of primary antibodies (anti-irisin antibody 1:500 and anti-GnRH 1:500) diluted in PBS containing 10\% normal goat serum, $0.05 \%$ TritonX-100 and 0.1\% BSA. Control sections were incubated in PBS with10\% normal goat serum, $0.05 \%$ TritonX-100 and 0.1\% BSA. Following incubation, sections were washed with PBS for $3 \times 15 \mathrm{~min}$ and incubated in a cocktail of secondary antibodies (Alexa Fluor 488 goat anti-rabbit 1:400 and Texas redconjugated goat anti-mouse IgG-TR 1:400) diluted in PBS containing 10\% normal goat serum, 0.05\% TritonX-100 and $0.1 \%$ BSA for $2 \mathrm{~h}$ in dark at room temperature $\left(25^{\circ} \mathrm{C}\right)$ on a shaker. Control sections were also incubated with secondary antibodies at this stage. After incubation, sections were again washed with PBS for $3 \times 15 \mathrm{~min}$. Later, all sections were mounted on super frosted glass slides and left overnight for drying in the dark at $4^{\circ} \mathrm{C}$ 
(Micro slides, Santa Cruz Biotechnology). Next day a coverslip was placed using laboratory-prepared Gelvatol as a mounting medium. Gelvatol was prepared by adding $10.5 \mathrm{~g}$ polyvinyl alcohol and few crystals of sodium azide in $12 \mathrm{~mL}$ glycerol. Then, $26 \mathrm{~mL}$ distilled water and $53 \mathrm{~mL}$ Tris ( $\mathrm{pH}$ 8.5) were added. The mixture was stirred with low heat for $6 \mathrm{~h}$ until reagents were properly dissolved. The mixture was placed overnight in a refrigerator and centrifuged at $5000 \boldsymbol{g}$ for $15 \mathrm{~min}$. Aliquots of Gelvatol were made and stored at $4^{\circ} \mathrm{C}$. Following mounting, slides were then left overnight for drying in dark at $4^{\circ} \mathrm{C}$ and stored until fluorescence microscopy.

\section{Real-time quantitative PCR}

For extraction of total RNA from hypothalamic, muscle and gonadal tissues (about $25 \mathrm{mg}$ ), a NucleoSpin RNA plus kit (Macherey-Nagel GmbH, Düren, Germany) was used according to the manufacturer's instructions. For the removal of genomic DNA from isolated RNA samples, a DNA-free DNA removal kit (AM1906, Ambion, Life Technologies) was used. Two micrograms of RNA was reverse transcribed into cDNA using the Omniscript RT kit 200 (Qiagen). The cDNA was diluted with deionized water to a final concentration of $15 \mathrm{ng} / \mu \mathrm{L}$ and stored at $-20^{\circ} \mathrm{C}$. For each sample, qPCR amplification was carried out in triplicates along with negative controls using the SybrGreen MasterMix (Applied Biosystems) and the StepOnePlus System quantitative PCR machine (Applied Biosystems) as previously described (Wahab et al. 2017). The sequence of all primers is given in Table 1 . These primers were tested by melting curve and efficiency check. The efficiencies of these primers were between 90 and $110 \%$. Due to stable expression, as compared to GAPDH and $A C T B$ in various developmental stages of the samples tested, the constitutively expressed 18sRNA transcripts were used as internal control for cDNA input. The $2^{-\Delta \Delta C T}$ method was used for relative mRNA levels calculation.

\section{Statistical analysis}

For the statistical comparison, the GraphPad Prism software (GraphPad Software Inc.) was used. For the statistical comparison between two groups, the Student's t-test was used, while the comparison between more than two groups was done with one-way ANOVA with post hoc Tukey test. The statistical significance level was set at $P \leq 0.05$ and is shown by different combinations of asterisks in the figures. All data are shown as means \pm s.E.M.

\section{Results}

FNDC5, ERRA and PGC1A transcript abundance in muscle and different compartments of the marmoset HPG axis

We compared FNDC5, ERRA and PGC1A mRNA abundance in hypothalamus, pituitary, gonad and muscle of female and male common marmoset monkeys (Fig. 1). As compared to hypothalamus and gonads in both female and male (Fig. 1A and B), FNDC5 mRNA abundance was significantly higher in muscle $(P<0.05)$. ERRA mRNA was expressed in hypothalamus and gonads at comparable levels in both sexes, while muscle ERRA mRNA expression levels were significantly $(P<0.05)$ higher than those in the hypothalamus and the gonads. PGC1A mRNA expression levels in the female muscle were much higher $(P<0.05)$

Table 1 List of primers used for real-time qPCR with Ensemble transcript ID/accession number.

\begin{tabular}{|c|c|c|c|}
\hline Gene & Transcript ID/Accession\# & Primer & Sequences $\left(5^{\prime}-3^{\prime}\right)$ \\
\hline FNDC5-Marmoset & ENSCJAT00000001944.1 & $\begin{array}{l}\text { Forward } \\
\text { Reverse }\end{array}$ & $\begin{array}{l}\text { ATCGTCGTGGTCCTGTTCAT } \\
\text { TCTGGTGTGCTGGTTTCTGA }\end{array}$ \\
\hline GAPDH-Marmoset & DD279474 & $\begin{array}{l}\text { Forward } \\
\text { Reverse }\end{array}$ & $\begin{array}{l}\text { TCGGAGTCAACGGATTTGGTC } \\
\text { TTCCCGTTCTCAGCCTTGAC }\end{array}$ \\
\hline PGC1A-Marmoset & ENSCJAG00000013024 & $\begin{array}{l}\text { Forward } \\
\text { Reverse }\end{array}$ & $\begin{array}{l}\text { AGCTTTCTGGGTGGACTCAA } \\
\text { CTGCTAGCAAGTTTGCCTCA }\end{array}$ \\
\hline ERRA-Marmoset & ENSCJAG00000018030 & $\begin{array}{l}\text { Forward } \\
\text { Reverse }\end{array}$ & $\begin{array}{l}\text { GCAAAGCCTTCTTCAAGA } \\
\text { TGATCTCACACTCATTGGA }\end{array}$ \\
\hline 18S rRNA-Marmoset & AB571241 & $\begin{array}{l}\text { Forward } \\
\text { Reverse }\end{array}$ & $\begin{array}{l}\text { CGCGGTTCTATTTTGTTGGT } \\
\text { AGTCGGGCATCGTTTATGGTC }\end{array}$ \\
\hline 18S rRNA-Mouse & AK135936.1 & $\begin{array}{l}\text { Forward } \\
\text { Reverse }\end{array}$ & $\begin{array}{l}\text { GGTTGATCCTGCCAGTAG } \\
\text { CGACCAAAGGAACCATAACT }\end{array}$ \\
\hline GnRH1-Mouse & ENSMUST00000111095.3 & $\begin{array}{l}\text { Forward } \\
\text { Reverse }\end{array}$ & $\begin{array}{l}\text { AGCACTGGTCCTATGGGTTG } \\
\text { GGGGTTCTGCCATTTGATCCA }\end{array}$ \\
\hline GAPDH-Mouse & ENSMUST00000117757.8 & $\begin{array}{l}\text { Forward } \\
\text { Reverse }\end{array}$ & $\begin{array}{l}\text { TGCACCACCAACTGCTTAG } \\
\text { GGATGCAGGGATGATGTTC }\end{array}$ \\
\hline
\end{tabular}



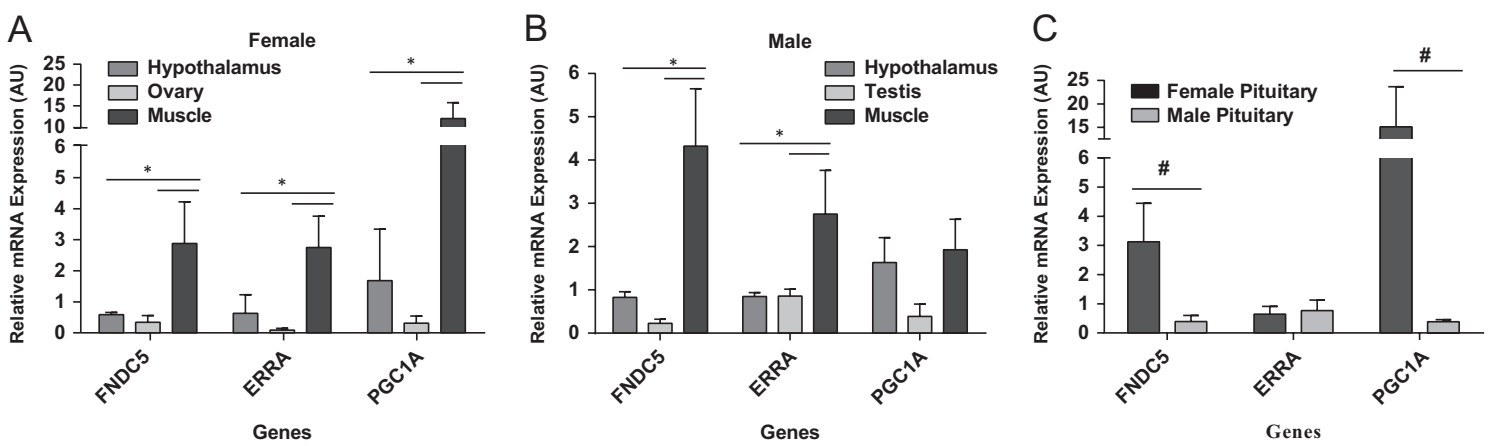

\section{Figure 1}

Comparison of FNDC5, ERRA and PGC1A expression in muscle and different compartments of the reproductive axis in the adult female and male common marmoset monkey. (A) In the female monkey, the transcript levels of FNDC5, ERRA and PGC1A were relatively high in muscle in comparison to ovary and hypothalamus. (B) In the male, the transcript abundance of FNDC5 and PGC1A were low in the testis as compared to muscle and hypothalamus. ERRA levels were high in muscle in comparison to both hypothalamus and testis. (C) The FNDC5 and PGC1A levels were high in female pituitary than male. There was no noteworthy difference in ERRA levels between male and female.

than those in the hypothalamus and the ovary. In contrast, in the male, muscle PGC1A levels were in the range of the male and also the female monkey hypothalamus. The relative PGC1A expression level in the female muscle was higher than that in the male muscle $(P<0.05)$. In the female pituitary, FNDC5 and PGC1A expression levels were higher as compared to male $(P<0.05)$ (Fig. 1C). There was no sex-specific difference in ERRA expression levels in the pituitary.

\section{Hypothalamic FNDC5, ERRA and PGC1A mRNA profiles at various stages of postnatal development}

We compared the relative levels of FNDC5, ERRA and $P G C 1 A$ in $\mathrm{NB}$, infantile, juvenile, pubertal and adult female (Fig. 2A, C and E) and male (Fig. 2B, D and $\mathrm{F})$ common marmoset monkey hypothalami. A significantly increased expression of FNDC5 mRNA relative to 18sRNA was observed in pubertal and adult tissues as compared to $\mathrm{NB}$, infantile and juvenile samples in both male and female $(P<0.05)$. FNDC5 mRNA levels were also significantly higher in the infantile stage as compared to NB in the male $(P<0.05)$, while no significant difference was detected in the present sample set between NB, infantile and juvenile stages in female monkey hypothalami.

There was a statistically significant $(P<0.05)$ increase in ERRA mRNA levels from the juvenile stage to the pubertal and adult stages in both female (Fig. 2C) and male (Fig. 2D). Like FNDC5, the transcript levels of ERRA were also significantly higher in infantile male monkeys in comparison to the NB. In summary, monkey hypothalamic ERRA transcript levels are strongly developmentally regulated peaking in the adult stage.
The hypothalamic PGC1A mRNA expression levels did not significantly vary between $\mathrm{NB}$, infantile and juvenile stages in both male and female monkeys (Fig. 2E and F). However, a statistically significant increase in PGC1A expression was detected from the juvenile to the adult stage in the female and from the juvenile to the pubertal stage in male marmoset hypothalami. Interestingly, in contrast to the females, in males, the PGC1A levels declined in adults compared to the pubertal stage. We conclude that there are sex-specific differences in the developmental hypothalamic expression profile of $P G C 1 \mathrm{~A}$ in the marmoset monkey.

\section{Irisin expression in marmoset and rhesus monkey hypothalamus}

Irisin was mainly detected in the ventromedial and the ARC of the adult hypothalamus (Fig. 2A). Very few weakly stained neurons were also noted in NB female monkey hypothalamus. No staining was observed in primary antibody-omitted or non-specific IgG-treated tissue sections (data not shown). Irisin and ERR $\alpha$ showed comparable developmental expression profiles (Fig. 2) suggesting an interdependence of both factors. Co-localization of cytoplasmic irisin (red signal) with nuclear ERR $\alpha$ (brown signal) was noted in a subset of neurons in the adult hypothalamus of the marmoset by IHC double-staining (Fig. 3B).

In order to provide first data on the potential role of irisin in the monkey hypothalamus, we analyzed in situ possible interactions of irisin-positive neurons with GnRH neurons. First, we determined the total number (mean \pm s.e.m.) of irisin-immunoreactive cell bodies observed in randomly selected sections from the ARC 


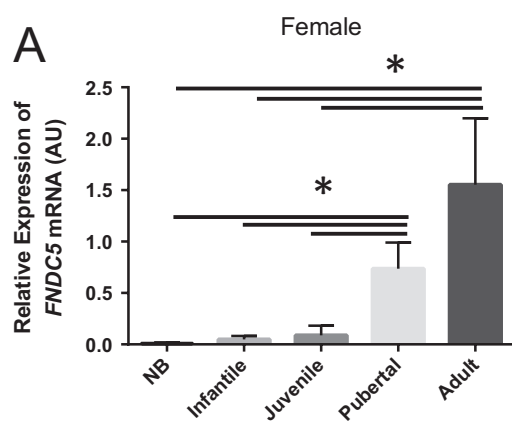

Postnatal Developmental Stages

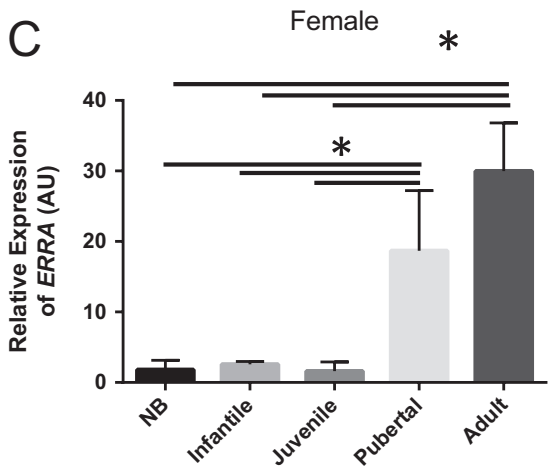

Postnatal Developmental Stages

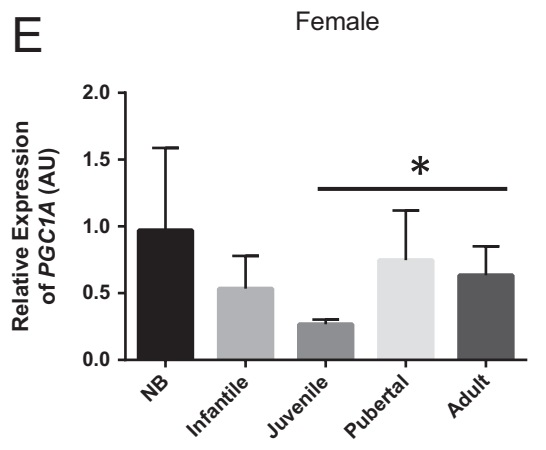

Postnatal Developmental Stages

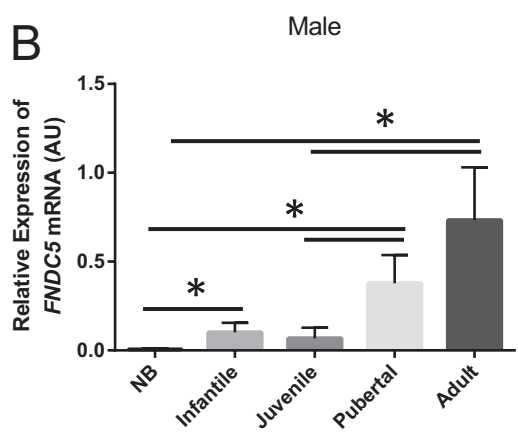

Postnatal Developmental Stages

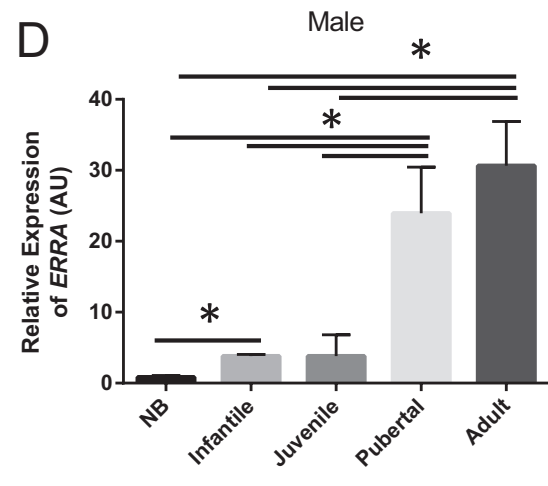

Postnatal Developmental Stages

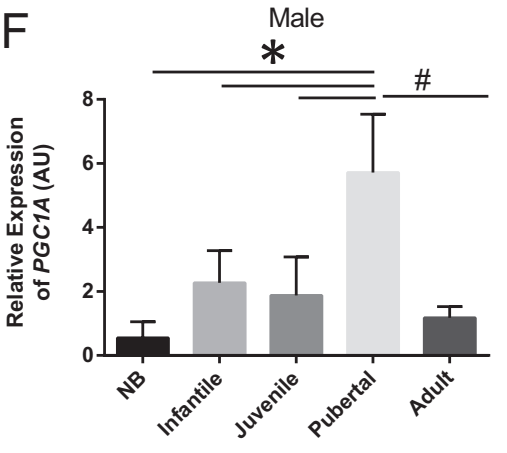

Postnatal Developmental Stages

\section{Figure 2}

Comparison of FNDC5, ERRA and PGC1A mRNA expression in hypothalami of female and male common marmoset at various postnatal developmental stages. (A and B) Hypothalamic FNDC5 mRNA expression significantly increased from the juvenile to the pubertal stages in both females and males. In male monkeys, a significant increase was also observed from NB to the infantile. FNDC5 transcript levels in hypothalami of both adult female and adult male marmosets were also significantly higher as compared to NB and juvenile stages. (C and D) Hypothalamic ERRA mRNA expression in female marmosets was significantly higher in pubertal and adult stages as compared to NB, infantile and juvenile stages. In male marmosets, hypothalamic ERRA expression significantly increased in infantile and pubertal/ adult marmosets as compared to NB and juvenile marmosets, respectively. ( $E$ and $F$ ) In female monkeys, a significant increase in PGC1A expression was detected only in adults as compared to juveniles, while there was no statistically significant difference compared to other stages in female marmosets. In male marmosets, pubertal PGC1A transcript levels were significantly higher as compared to NB, infantile, juvenile and adult. Values are expressed as the mean \pm S.E.M. ( ${ }^{*} P<0.05$ significant increase, $\# P<0.05$ significant decrease). region in the adult male rhesus monkeys, which were subjected to 48 -h fasting $(n=2)$ and normal feeding $(n=2)$. Irisin-immunoreactive cell bodies were significantly $\left({ }^{\star} \mathrm{P}<0.05\right)$ increased in monkeys deprived of food for $48 \mathrm{~h}$ as compared to those monkeys which were fed ad libitum (Fig. 3C and D). Possible (synaptic) contacts (yellow) between irisin-immunoreactive fibers (green) and GnRHimmunoreactive cell bodies (red) in the ARC of the $\mathrm{MBH}$ hypothalamic area of the normal fed adult male rhesus monkeys were noted (Fig. 3E). Approximately, 5\% of the GnRH-positive neurons received putative contacts from irisin-positive cells. Altogether, these data suggest (i) an induction of irisin in the rhesus monkey hypothalamus by fasting and (ii) although rare but a putative interaction between irisin and GnRH neurons.
Expression profiles of FNDC5, ERRA and PGC1A in the anterior and posterior hypothalamus of neonatal and adult monkeys

The transcript abundances of FDNC5 were significantly increased $(P<0.05-0.01)$ in both the anterior and posterior hypothalamus of the adult common marmosets as compared to NB monkeys in both sexes (Fig. 4A and B). Moreover, the abundances of FNDC 5 mRNA in the adult posterior as well as in the anterior hypothalamus were also significantly higher in female as compared to male in these hypothalamic areas. The abundance of ERRA mRNA levels was significantly higher in the adult female marmoset monkey in both compartments as compared to the NB stage (Fig. 4C and D). Although an 


\begin{tabular}{l|l|l|r|r|} 
Journal of & F Wahab et al. & Irisin in the hypothalamus & $\mathbf{2 4 1 : 3}$ & 182 \\
Endocrinology &
\end{tabular}

A

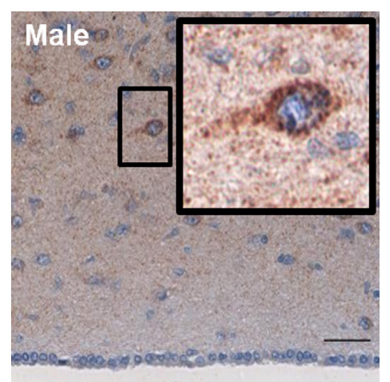

C

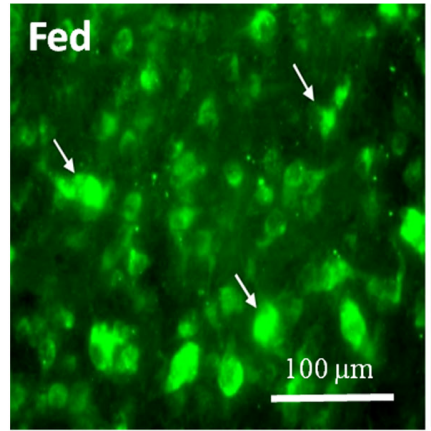

E

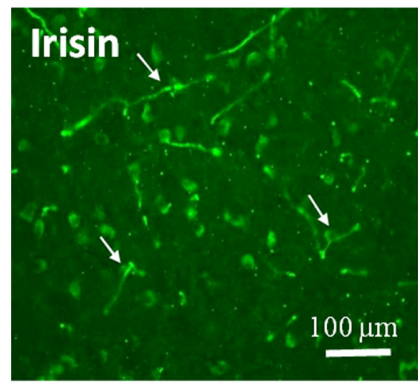

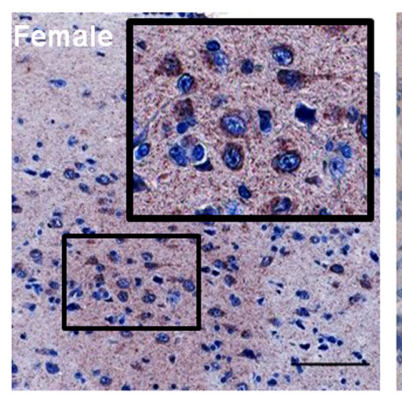

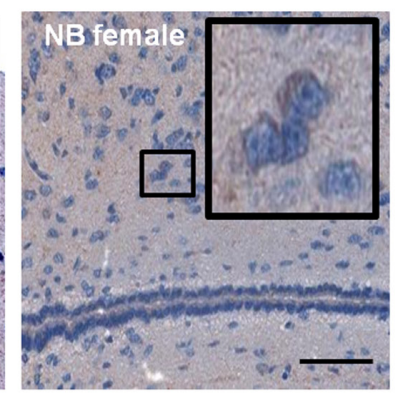

D

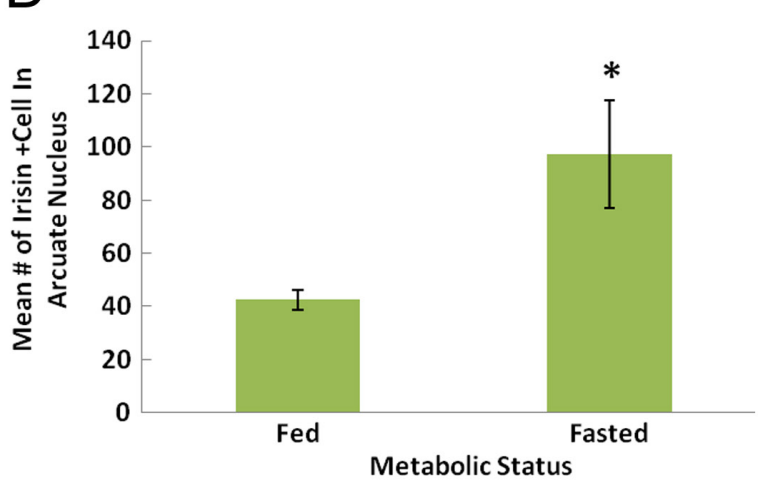

Figure 3

IHC analysis of irisin expression in the marmoset monkey hypothalami (A and B) and irisin expression and putative interaction with GnRH neurons in the rhesus monkey $\mathrm{MBH}$ ( $\mathrm{C}$ and $\mathrm{E})$. (A) Photographs showing irisin immunostaining in common marmoset monkey hypothalami. Clear immunostaining of irisin was observed in the ARC and the ventromedial nucleus of the hypothalamus of both male and female monkeys. There were very few irisin-expressing cells in NB female hypothalamus. No staining was observed in IgG isotype-incubated tissue section (figure not shown). Magnification is $40 \times$ and $100 \mu$ m scale bars. (B) Immunohistochemical double-staining of ERR $\alpha$ and irisin. Nuclear ERR $\alpha$ is shown in brown and cytoplasmic irisin in red. (C) Photomicrographs showing irisin-immunoreactive cell bodies in the ARCs of adult male rhesus monkeys in fed and fasted states. Magnification is $40 \times$ and $100 \mu m$ scale bars. (D) Comparison of mean \pm S.E.M. number of irisin-immunoreactive cell bodies in the ARC in adult male rhesus monkeys which were subjected to 48 -h fasting $(n=2)$ and normal feeding $(n=2)$. Irisin-immunoreactive cell bodies were significantly ( $* P<0.05)$ increased in monkeys deprived of food for $48 \mathrm{~h}$ as compared to those monkeys which were fed ad libitum. (E) Photomicrographs showing putative interaction of irisin-immunoreactive fibers and GnRHimmunoreactive cell bodies in the $\mathrm{MBH}$ hypothalamic area of a normal fed adult male rhesus monkeys. Arrowheads show the immunoreactive fibers and cell bodies at 20x magnification. Putative synaptic contact (yellow color) between irisin fibers (green) and GnRH cell body (red) was observed.

increasing trend was observed in the male, no significant change was detected at the current sample size. Also, no significant difference was observed at the current samples size between the PGC1A transcript levels of $\mathrm{NB}$ and adult female and male monkeys in the anterior hypothalamus (Fig. 4E and F). In contrast, there was a significant difference in the posterior hypothalamus in both, adult male and female as compared to NB. Additionally, there was a sex-specific difference: the EERA and PGC1A transcript levels of female monkeys in the posterior hypothalamus were significantly higher than those in adult males. These data show that these genes are tissue- and sex-specifically expressed in the neonatal and adult monkey. The adult female 


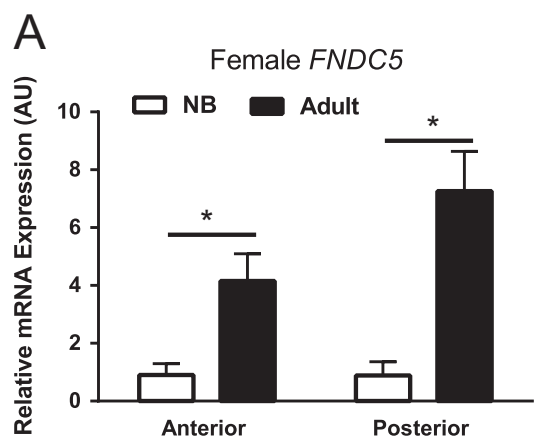

Parts of Hypothalamus

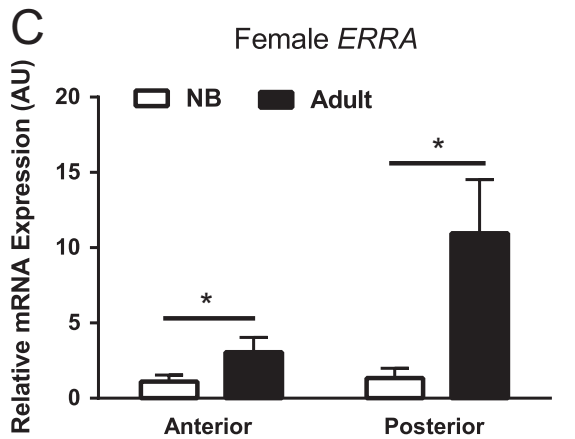

Parts of Hypothalamus

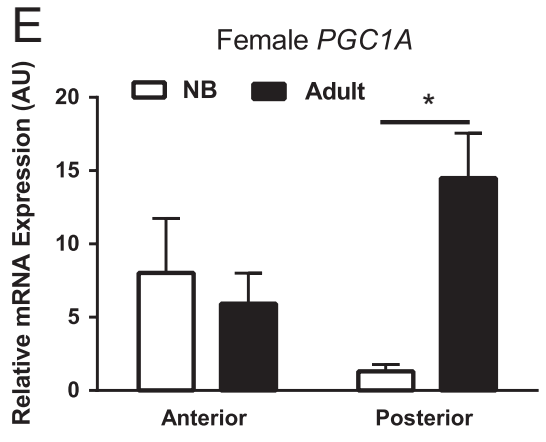

Parts of Hypothalamus

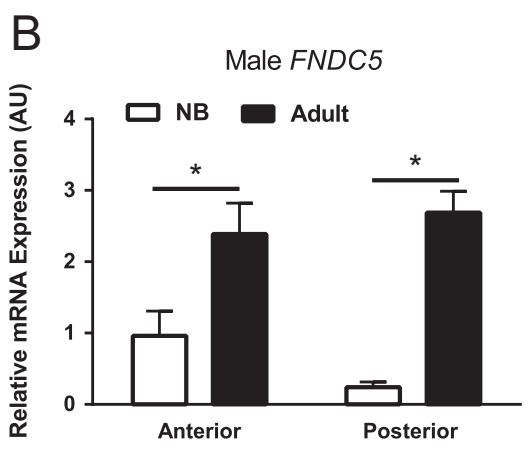

Parts of Hypothalamus
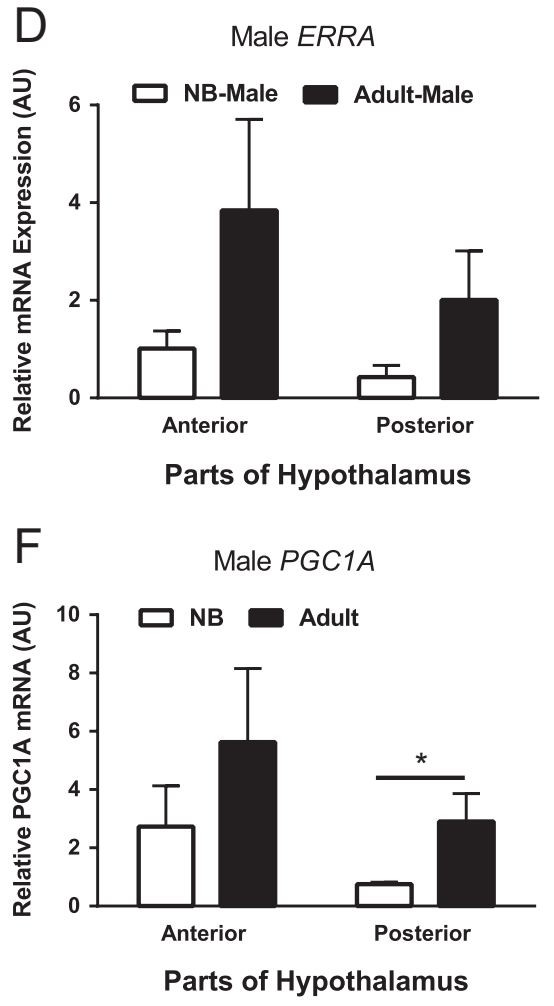

Figure 4

Expression levels of FNDC5, ERRA and PGC1A mRNA in anterior and posterior hypothalami in NB and adult female and male marmoset monkeys. (A and B) FNDC5 transcript levels in both female (A) and male (B) adult common marmosets significantly increased in the anterior as well as in the posterior hypothalamus as compared to NB. (C and D) ERRA mRNA expression was significantly heightened in the anterior and posterior hypothalamus only in female adult monkeys as compared to NB. There was no significant difference between NB and adult ERRA levels in male monkeys. ( $E$ and $F$ ) There was no statistically significant difference in $P G C 1 A$ $m R N A$ levels in anterior hypothalami between NB and adult stages of male and female monkeys. In posterior hypothalami, $P G C 1 A$ gene expression was significantly $\left({ }^{*} P<0.05-0.01\right)$ higher in adult female and male monkeys as compared to female and male NB, respectively. posterior hypothalamus showed the highest relative expression levels for all genes tested in the current study.

\section{Irisin regulates $\mathrm{GnRH}$ expression in a mouse hypothalamic neuronal cell line}

In order to test hypothalamic irisin action (i) in a defined system and (ii) in the absence of a primate hypothalamic neuronal cell line and (iii) in the absence of a sufficient number of primary tissues for functional neuroendocrine analyses, we tested the effect of irisin on Gnrh1 mRNA expression in the well-established mouse GT1-7 hypothalamic neuronal cell line. We collected samples after 15 and $60 \mathrm{~min}$ of irisin application (Fig. 5). $10 \mathrm{nM}$ concentration of irisin significantly increased the expression of Gnrh1 transcripts levels at both time points. A trend toward increased Gnrh1 transcript levels was already noted for the $1 \mathrm{nM}$ concentration of irisin. We tested also the release of GnRH from GT1-7 cells after $60 \mathrm{~min}$. $10 \mathrm{nM}$ of irisin caused a significant $(P<0.03)$ increase in $\mathrm{GnRH}$ concentration in the cell culture medium as compared to control (PBS alone instead of irisin dissolved in PBS). We conclude that irisin positively regulates hypothalamic Gnrh1 transcript levels and, even more pronounced, GnRH release from the mouse GT1-7 cell line.

\section{Discussion}

The HPG axis is a control unit integrating molecular signaling originating from and received by the 

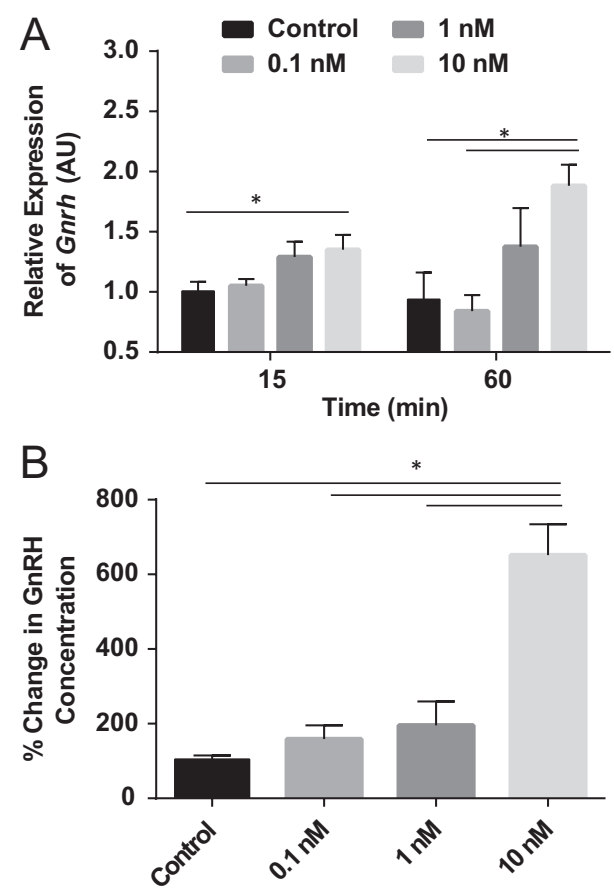

Irisin Concentration

\section{Figure 5}

Irisin effect on Gnrh1 expression and GnRH release from the mouse hypothalamic neuronal cell line GT1-7. (A) $10 \mathrm{nM}$ concentration of irisin caused a significant increase in Gnrh1 expression in GT1-7 hypothalamic line at both, 15 and $60 \mathrm{~min}$. (B) Likewise, $10 \mathrm{nM}$ concentration of irisin also caused a significant increase in $\mathrm{GnRH}$ concentration after $1 \mathrm{~h}$ of application.

hypothalamus, the pituitary and the gonads. They all are critical for the regulation of reproductive function (Terasawa \& Fernandez 2001, Clarke 2011, Plant 2015a). Some of these molecular signals constitute stimulating loops for reproductive function, while others lead to inhibitory loops (Terasawa \& Fernandez 2001, Clarke 2011, Plant 2015a). A very recent potential candidate for the modulation of reproductive function is irisin (Wahab et al. 2016b, Poretsky et al. 2017). In this study, we characterized developmental expression profiles of irisin and two of its regulators in the NHP hypothalamus and showed irisin expression in different components of the monkey HPG axis. To initially characterize the expression of FNDC5 along the HPG axis, transcript abundance of FNDC5 along with PGC1a and ERRA, which have been shown to regulate or modulate peripheral and central irisin expression (Boström et al. 2012, Wrann et al. 2013), were checked in hypothalamus, pituitary, ovary and testis. Subsequently, the quantification of FNDC5, ERRA and PGC1A mRNA levels was carried out in hypothalami of common marmoset monkeys at neonatal, infantile, juvenile, pubertal and adult stages for checking alterations in FNDC5 expression at various postnatal developmental stages in a non-human primate. A robust increase in the expression of FNDC5 and ERRA transcript were noted from the juvenile to the pubertal stage. These parallel profiles of both transcripts may suggest regulation of irisin by the transcription factor ERR $\alpha$, as has been described before (Wrann et al. 2013). This speculation was corroborated by the finding of co-expression of ERRo with irisin in individual hypothalamic neurons.

The HPG axis exhibits three defined periods of activity during prenatal and postnatal development in primates including human: the first during the fetal life, the second during the first postnatal months (minipuberty), and a third at the onset of (true) puberty (Kuiri-Hänninen et al. 2014). The mechanism of both, postnatal reactivation of the HPG axis and in particular of true puberty onset is still poorly understood. Different hypotheses are proposed for puberty onset (Baker 1985, Terasawa \& Fernandez 2001, Plant 2015b). According to one of the hypotheses, a critical body weight (fat and protein mass) is required for pubertal activation of the HPG axis (Baker 1985). At the onset of puberty, the hypothalamic neuronal circuitry important for the regulation of reproduction is reactivated when the body weight (body fat) reached a particular level that is crucial for puberty onset and supporting adult reproduction (Kennedy \& Mitra 1963, Frisch \& Revelle 1970, Frisch \& McArthur 1974, Baker 1985, Terasawa et al. 2012). When the body reaches this critical fat and/or muscular mass, a metabolic signal(s) communicates this information to the hypothalamic neuronal network governing puberty onset. However, the nature of this metabolic trigger is still not clear. Our data from this study suggest that irisin could be a player in the pubertal activation of the HPG axis. Nevertheless, whether irisin, in fact, serves as a relevant metabolic signal which may link body fat/ muscular mass with the hypothalamic neurohormonal network governing puberty onset and adult reproduction needs deeper in vivo investigations. Importantly, however, plasma levels of irisin have been reported to change during various stages of puberty (Hofmann et al. 2014, Reinehr et al. 2015). Moreover, we observed that the abundance of fibronectin type III domain-containing-5 (FNDC5) transcripts increase in the hypothalamus of both male and female monkeys around the time of puberty onset. These data suggest that irisin might be involved in the regulation of pubertal activation of the HPG axis.

We have noted irisin expression mainly in the ventromedial nucleus and the ARC of the hypothalamus in marmoset and rhesus monkeys. These regions of the hypothalamus are involved in the regulation of feeding, 
energy homeostasis and reproduction (Wynne et al. 2005, Wahab et al. 2013). Importantly, we observed direct contact of irisin-immunoreactive fibers and with GnRH neurons. Whether irisin directly or indirectly modulates synthesis and release of GnRH requires proper investigation. Moreover, studies on the role of PGC- $1 \alpha$ and ERR $\alpha$, which have been shown to regulate/modulate peripheral irisin expression, in the regulation of central hypothalamic irisin expression will also be important. Further studies on the co-localization of irisin with neuropeptides expressed in the hypothalamic ventromedial and the arcuate nucleus and single cell transcriptomics (Chen et al. 2017) are also of relevance.

We detected sex-specific differences in the expression of FNDC5 and PGC1A in the pituitary, muscle and posterior hypothalamus. The FNDC5 and PGC1A transcript levels were significantly higher in female muscle, pituitary and posterior hypothalamus than in the corresponding male tissues. Previously, another study in human also reported a higher level of PGC1A in female muscle than in male (Maher et al. 2009). Moreover, there was also a sex-specific difference in the EERA in the posterior hypothalamus. The transcript levels of ERRA in the posterior hypothalamus of female monkeys were significantly higher than those in adult males. These data show for the first time that these genes are tissue- and sex-specifically expressed in the neonatal and adult monkey. However, whether they play any role in the sex-specific differences in endocrine physiology needs scholarly investigations.

GnRH is a central regulator of the endocrine control of gametogenesis (Clarke 2011). Here, we provide data that indicate that irisin neurons may directly contact GnRH neurons in the rhesus monkey hypothalamus. This observation is supported by the fact that irisin mildly enhanced the transcript level abundance of GnRH in the mouse GT1-7 cell line after $1 \mathrm{~h}$ of irisin action. An extended incubation period and/or an increased concentration of irisin may further increase the GnRH abundance. Moreover, irisin led to a strong release of GnRH from the GT1-7 cell line after $1 \mathrm{~h}$, when added in a $10 \mathrm{nM}$ concentration. In fact, we detected a statistically significant six-fold increase under this condition. Together, these data suggest that irisin may modulate GnRH expression and show that it is involved in the release of GnRH from the GT1-7 cell line. Future in vivo experiments has to confirm our in vitro findings.

In summary, our results demonstrate a significant increase in the abundance of FNDC5 during infantile and postpubertal stages compared to the respective preceding developmental stages in a non-human primate species.
Irisin neurons putatively interact with GnRH neurons in rhesus monkey hypothalamus, and irisin is also involved in the regulation of GnRH release from mouse hypothalamic neurons. These findings, together with already available literature (Poretsky et al. 2017), suggest a potential role of irisin signaling in the regulation of the reproductive axis during puberty in primates. Nevertheless, more detailed studies including functional and mechanistic experiments are needed to further characterize the role and hierarchical position of irisin in the molecular cascade regulating the neuroendocrine reproductive axis in primates.

\section{Declaration of interest}

The authors declare that there is no conflict of interest that could be perceived as prejudicing the impartiality of the research reported.

\section{Funding}

The research presented here was partly funded by a grant from the Alexander von Humboldt Foundation (AvH), Germany. FW was a Georg Forster Fellow of the AvH. The research work in RB's laboratory is supported by the German Primate Center, which is a Leibniz Institute financed by the Federal Republic of Germany and the federal states.

\section{Acknowledgements}

The authors highly appreciate technical assistance from Nicole Umland.

\section{References}

Al-Gubory KH, Hervieu J \& Fowler PA 2003 Effects of pregnancy on pulsatile secretion of LH and gonadotrophin-releasing hormoneinduced LH release in sheep: a longitudinal study. Reproduction $\mathbf{1 2 5}$ 347-355. (https://doi.org/10.1530/rep.0.1250347)

Aydin S, Kuloglu T, Aydin S, Kalayci M, Yilmaz M, Cakmak T, Albayrak S, Gungor S, Colakoglu N \& Ozercan IH 2014 A comprehensive immunohistochemical examination of the distribution of the fatburning protein irisin in biological tissues. Peptides 61 130-136. (https://doi.org/10.1016/j.peptides.2014.09.014)

Baker ER 1985 Body weight and the initiation of puberty. Clinical Obstetrics and Gynecology 28 573-579. (https://doi. org/10.1097/00003081-198528030-00013)

Barja-Fernández S, Folgueira C, Castelao C, Al-Massadi O, Bravo SB, GarciaCaballero T, Leis R, Pardo M, Casanueva FF \& Seoane LM 2016 FNDC5 is produced in the stomach and associated to body composition. Scientific Reports 6 23067. (https://doi.org/10.1038/srep23067)

Beshay VE \& Carr BR 2013 Hypothalamic-pituitary-ovarian axis and control of the menstrual cycle. In Clinical Reproductive Medicine and Surgery, pp 31-42. Eds T Falcone \& WW Hurd. New York, NY, USA: Springer. (https://doi.org/10.1007/978-1-4614-6837-0_2)

Boström P, Wu J, Jedrychowski MP, Korde A, Ye L, Lo JC, Rasbach KA, Boström EA, Choi JH, Long JZ, et al. 2012 A PGC1- $\alpha$-dependent myokine that drives brown-fat-like development of white fat and thermogenesis. Nature $\mathbf{4 8 1}$ 463-468. (https://doi.org/10.1038/ nature10777) 
Castellano JM \& Tena-Sempere M 2016 Metabolic control of female puberty: potential therapeutic targets. Expert Opinion on Therapeutic Targets 20 1181-1193. (https://doi.org/10.1080/14728222.2016.121 2015)

Chen R, Wu X, Jiang L \& Zhang Y 2017 Single-cell RNA-Seq reveals hypothalamic cell diversity. Cell Reports 18 3227-3241. (https://doi. org/10.1016/j.celrep.2017.03.004)

Clarke IJ 2011 Control of GnRH secretion: one step back. Frontiers in Neuroendocrinology 32 367-375. (https://doi.org/10.1016/j. yfrne.2011.01.001)

Clarke IJ \& Caraty A 2013 Kisspeptin and seasonality of reproduction. Advances in Experimental Medicine and Biology 784 411-430. (https:// doi.org/10.1007/978-1-4614-6199-9_19)

Corradi PF, Corradi RB \& Greene LW 2016 Physiology of the hypothalamic pituitary gonadal axis in the male. Urologic Clinics of North America 43 151-162. (https://doi.org/10.1016/j.ucl.2016.01.001)

Crown A, Clifton DK \& Steiner RA 2007 Neuropeptide signaling in the integration of metabolism and reproduction. Neuroendocrinology $\mathbf{8 6}$ 175-182. (https://doi.org/10.1159/000109095)

Dun SL, Lyu RM, Chen YH, Chang JK, Luo JJ \& Dun NJ 2013 Irisin-immunoreactivity in neural and non-neural cells of the rodent. Neuroscience 240 155-162. (https://doi.org/10.1016/j. neuroscience.2013.02.050)

Evans MC \& Anderson GM 2017 Neuroendocrine integration of nutritional signals on reproduction. Journal of Molecular Endocrinology 58 R107-R128. (https://doi.org/10.1530/JME-16-0212)

Frisch RE \& McArthur JW 1974 Menstrual cycles: fatness as a determinant of minimum weight for height necessary for their maintenance or onset. Science 185 949-951. (https://doi.org/10.1126/ science.185.4155.949)

Frisch RE \& Revelle R 1970 Height and weight at menarche and a hypothesis of critical body weights and adolescent events. Science 169 397-399. (https://doi.org/10.1126/science.169.3943.397)

Henningsen JB, Gauer F \& Simonneaux V 2016 RFRP neurons - the doorway to understanding seasonal reproduction in mammals. Frontiers in Endocrinology 7 36. (https://doi.org/10.3389/ fendo.2016.00036)

Hofmann T, Elbelt U \& Stengel A 2014 Irisin as a muscle-derived hormone stimulating thermogenesis - a critical update. Peptides 54 89-100. (https://doi.org/10.1016/j.peptides.2014.01.016)

Kennedy GC \& Mitra J 1963 Body weight and food intake as initiating factors for puberty in the rat. Journal of Physiology $166408-418$. (https://doi.org/10.1113/jphysiol.1963.sp007112)

Kim H, Wrann CD, Jedrychowski M, Vidoni S, Kitase Y, Nagano K, Zhou C, Chou J, Parkman V-JA, Novick SJ, et al. 2018 Irisin mediates effects on bone and fat via $\alpha \mathrm{V}$ integrin receptors. Cell 175 1756-1768. e17. (https://doi.org/10.1016/j.cell.2018.10.025)

Kuiri-Hänninen T, Sankilampi U \& Dunkel L 2014 Activation of the hypothalamic-pituitary-gonadal axis in infancy: minipuberty. Hormone Research in Paediatrics 82 73-80. (https://doi. org/10.1159/000362414)

Maher AC, Fu MH, Isfort RJ, Varbanov AR, Qu XA \& Tarnopolsky MA 2009 Sex differences in global mRNA content of human skeletal muscle. PLoS One 4 e6335. (https://doi.org/10.1371/journal. pone.0006335)

Mellon PL, Windle JJ, Goldsmith PC, Padula CA, Roberts JL \& Weiner RI 1990 Immortalization of hypothalamic GnRH neurons by genetically targeted tumorigenesis. Neuron 5 1-10. (https://doi.org/10.1016/08966273(90)90028-E)

Palazzi X \& Bordier N 2008 The marmoset brain in stereotaxic coordinates. In The Marmoset Brain in Stereotaxic Coordinates, pp 1-59. Eds X Palazzi \& N Bordier. New York, NY, USA: Springer. (https://doi. org/10.1007/978-0-387-78385-7_1)

Perakakis N, Triantafyllou GA, Fernández-Real JM, Huh JY, Park KH, Seufert J \& Mantzoros CS 2017 Physiology and role of irisin in glucose homeostasis. Nature Reviews: Endocrinology 13 324-337. (https://doi. org/10.1038/nrendo.2016.221)

Plant TM 2015a 60 YEARS OF NEUROENDOCRINOLOGY: The hypothalamo-pituitary-gonadal axis. Journal of Endocrinology 226 T41-T54. (https://doi.org/10.1530/JOE-15-0113)

Plant TM $2015 b$ Neuroendocrine control of the onset of puberty. Frontiers in Neuroendocrinology 38 73-88. (https://doi.org/10.1016/j. yfrne.2015.04.002)

Poretsky L, Islam J, Avtanski D, Lin YK, Shen YL, Hirth Y, Lesser M, Rosenwaks Z \& Seto-Young D 2017 Reproductive effects of irisin: initial in vitro studies. Reproductive Biology 17 285-288. (https://doi. org/10.1016/j.repbio.2017.05.011)

Reinehr T, Elfers C, Lass N \& Roth CL 2015 Irisin and its relation to insulin resistance and puberty in obese children: a longitudinal analysis. Journal of Clinical Endocrinology and Metabolism 100 2123-2130. (https://doi.org/10.1210/jc.2015-1208)

Roca-Rivada A, Castelao C, Senin LL, Landrove MO, Baltar J, Crujeiras AB, Seoane LM, Casanueva FF \& Pardo M 2013 FNDC5/ Irisin is not only a Myokine but also an adipokine. PLoS One $\mathbf{8}$ e60563. (https://doi.org/10.1371/journal.pone.0060563)

Rodríguez A, Becerril S, Ezquerro S, Méndez-Giménez L \& Frühbeck G 2016 Crosstalk between adipokines and myokines in fat browning Acta Physiologica 219 362-381. (https://doi.org/10.1111/ apha.12686)

Terasawa E \& Fernandez DL 2001 Neurobiological mechanisms of the onset of puberty in primates. Endocrine Reviews 22 111-151. (https:// doi.org/10.1210/edrv.22.1.0418)

Terasawa E, Kurian JR, Keen KL, Shiel NA, Colman RJ \& Capuano SV 2012 Body weight impact on puberty: effects of high-calorie diet on puberty onset in female rhesus monkeys. Endocrinology 153 1696-1705. (https://doi.org/10.1210/en.2011-1970)

Wade GN \& Jones JE 2004 Neuroendocrinology of nutritional infertility. American Journal of Physiology: Regulatory, Integrative and Comparative Physiology 287 R1277-R1296. (https://doi.org/10.1152/ ajpregu.00475.2004)

Wahab F, Quinton R \& Seminara SB 2011a The kisspeptin signaling pathway and its role in human isolated GnRH deficiency. Molecular and Cellular Endocrinology 346 29-36. (https://doi.org/10.1016/j. mce.2011.05.043)

Wahab F, Ullah F, Chan YM, Seminara SB \& Shahab M $2011 b$ Decrease in hypothalamic Kiss1 and Kiss1r expression: a potential mechanism for fasting-induced suppression of the HPG axis in the adult male rhesus monkey (Macaca mulatta). Hormone and Metabolic Research 43 81-85. (https://doi.org/10.1055/s-0030-1269852)

Wahab F, Atika B \& Shahab M 2013 Kisspeptin as a link between metabolism and reproduction: evidences from rodent and primate studies. Metabolism: Clinical and Experimental 62 898-910. (https:// doi.org/10.1016/j.metabol.2013.01.015)

Wahab F, Shahab M \& Behr R 2015 The involvement of gonadotropin inhibitory hormone and kisspeptin in the metabolic regulation of reproduction. Journal of Endocrinology 225 R49-R66. (https://doi. org/10.1530/JOE-14-0688)

Wahab F, Atika B, Shahab M \& Behr R 2016a Kisspeptin signalling in the physiology and pathophysiology of the urogenital system. Nature Reviews: Urology 13 21-32. (https://doi.org/10.1038/ nrurol.2015.277)

Wahab F, Shahab M \& Behr R $2016 b$ Hypothesis: irisin is a metabolic trigger for the activation of the neurohormonal axis governing puberty onset. Medical Hypotheses 95 1-4. (https://doi.org/10.1016/j. mehy.2016.08.003)

Wahab F, Drummer C, Schlatt S \& Behr R 2017 Dynamic regulation of hypothalamic DMXL2, KISS1, and RFRP expression during postnatal development in non-human primates. Molecular Neurobiology 54 8447-8457. (https://doi.org/10.1007/ s12035-016-0329-x) https://joe.bioscientifica.com

https://doi.org/10.1530/JOE-18-0574 (c) 2019 Society for Endocrinology Published by Bioscientifica Ltd. Printed in Great Britain 
Wahab F, Atika B, Ullah F, Shahab M \& Behr R 2018 Metabolic impact on the hypothalamic kisspeptin-Kiss1r signaling pathway. Frontiers in Endocrinology 9 123. (https://doi.org/10.3389/fendo.2018.00123)

Winter JS, Ellsworth L, Fuller G, Hobson WC, Reyes FI \& Faiman C 1987 The role of gonadal steroids in feedback regulation of gonadotropin secretion at different stages of primate development. Acta Endocrinologica 114 257-268. (https://doi.org/10.1530/acta.0.1140257)
Wrann CD, White JP, Salogiannnis J, Laznik-Bogoslavski D, Wu J, Ma D, Lin JD, Greenberg ME \& Spiegelman BM 2013 Exercise induces hippocampal BDNF through a PGC-1 $\alpha /$ FNDC5 pathway. Cell Metabolism 18 649-659. (https://doi.org/10.1016/j.cmet.2013.09.008)

Wynne K, Stanley S, McGowan B \& Bloom S 2005 Appetite control. Journal of Endocrinology 184 291-318. (https://doi.org/10.1677/ joe.1.05866)

Received in final form 19 March 2019

Accepted 26 March 2019

Accepted Preprint published online 26 March 2019 (c) 2019 Society for Endocrinology Published by Bioscientifica Ltd.
Printed in Great Britain 